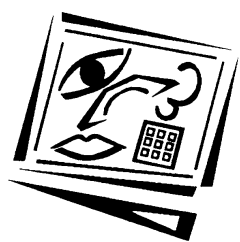

\title{
Scaffolding and integrated assessment in computer assisted learning (CAL) for children with learning disabilities
}

\author{
Ivan L. Beale \\ University of New South Wales
}

\begin{abstract}
Computer assisted learning (CAL) can involve a computerised intelligent learning environment, defined as an environment capable of automatically, dynamically and continuously adapting to the learning context. One aspect of this adaptive capability involves automatic adjustment of instructional procedures in response to each learner's performance, to facilitate the ease of learning and to minimise errors during learning. This process of dynamically varying the help provided to the learner by the instructor has been termed scaffolding. A bonus from using scaffolding is that the programming algorithms by which scaffolding is achieved allow integrated assessment of the learner's performance. This paper outlines the nature and origins of scaffolding concepts and illustrates their application as instructional design strategies in an experimental intelligent learning environment designed to teach basic reading skills to children with learning disabilities. The paper also illustrates the role of integrated assessment as an essential component of scaffolding and as a means of monitoring and recording the learning process.
\end{abstract}

\section{Introduction}

As personal computers have become more powerful, cheaper and multimedia oriented, there has been a rapidly growing interest in their potential as a vehicle for computer assisted learning (CAL). Emerging from this interest is the concept of the intelligent learning environment, defined as an environment capable of automatically, dynamically and continuously adapting to the learning context (Sampson, Karagiannidis \& Kinshuk, 2002). One aspect of this adaptive capability involves automatic dynamic adjustment of instructional procedures to facilitate the ease of learning and to minimise errors during learning. This adjustment process individualises the instructional process in response to information provided by the behaviour of the individual learner in the learning environment. If the 
system detects that the task is difficult for the learner, the task is altered to make it easier. If the system detects that the learner is becoming more proficient, the task is altered to make it more difficult. By interactively adjusting task difficulty, the learner is assisted to reach the desired learning goal along an individually tailored path.

When used by human tutors rather than computers, this process of dynamically varying the help provided to the learner by the instructor has been associated with the metaphor of 'scaffolding'. This idea is often attributed to Vygotsky, although the actual metaphor was first proposed by Wood and colleagues (Wood, Bruner \& Ross, 1976) and more recently has been refined by others (Lepper, Drake \& O'Donnell-Johnson, 1997). Scaffolding has sometimes been confused with the related concept of 'support', but is different in that it implies the removal and reinstatement of support according to the learner's need.

Scaffolding has become widely used in education as a metaphor for the use of additional elements that are added to a basic instructional format to facilitate learning and are later removed when the learner no longer requires them. Simple examples of scaffolding components are: a dot temporarily placed on the left of a printed sentence to cue a beginning reader where to start; hyphens used temporarily to separate syllables in polysyllabic words to assist the reader to sound out the words; and, providing progressively delayed prompts to assist a learner to supply an answer to a question. Scaffolding may be seen as beneficial for learning if it has one or more of the following effects: reduces the amount of instruction needed to reach a learning goal; reduces the number of errors made during learning; or, reduces frustration and other negative emotional responses to learning difficulties.

Although scaffolding has been widely recommended (and debated) for use by human teachers (Butler, 1998; Lepper et al., 1997; Scruggs \& Mastropieri, 1998; Wong, 1998), there is little published information about scaffolding strategies for intelligent learning environments used in CAL (Denning \& Smith, 1998; Dube, Iennaco, Rocco, Klederas, \& McIlvane, 1992). The purpose of this paper is to briefly review behavioural research on scaffolding principles and to illustrate the application of these principles in an intelligent learning environment developed to teach basic reading skills to children with learning disabilities. The paper also demonstrates how integrated assessment of the learner's performance is an essential component of scaffolding algorithms and can be used to provide assessment information to both the learner and the teacher on an ongoing basis. 


\section{Behavioural concepts that contribute to the development of scaffolding principles}

It has been noted that attempts by human teachers to use scaffolding strategies may be ineffective or even counterproductive unless the teacher has a clear understanding of the learning principles underlying the concept (Reid, 1998). Integrating these principles to generate effective scaffolding strategies does require some knowledge and skill. It is also necessary that teachers be able to design strategies themselves, since for many learning contexts there are no detailed guidelines to follow and often very little prior research that can be relied on (Lepper et al., 1997). Digital interactive multimedia have been seen as an ideal vehicle for intelligent learning environments using scaffolding strategies. For example, both scaffolding and integrated (continuous) assessment have been listed among the desiderata for future universally designed learning environments (Hitchcock, 2001).

Unfortunately, educational software designers have done little as yet to apply evidence based scaffolding principles in their products, even though teachers apparently would like access to software that is $\mathrm{s}$ responsive to variation in learners' ability levels (Judge, 2001). Because most analyses of scaffolding and handbooks describing applications have been focused on the strategies used by human tutors (Lepper et al., 1997; Wolery, Ault, \& Doyle, 1992), it would be useful to have analyses and applications that are focused on the design of digital learning environments.

Summarised below are some of the learning concepts from the behavioural research literature that are relevant to the design of scaffolding components of intelligent learning environments. Although these concepts arise from research in the behaviourist/instructivist paradigm, potentially they can play a useful part within all learning environments, including those based on a constructivist model, provided only that there are clear behavioural goals that can be set for the learner to achieve. Especially in the case where software applications are used to help children with learning difficulties learn specific skills that are not negotiable in standard school curricula, for example letter recognition, instructional approaches to learning will continue to have an important place (Kameenui, Simmons, Chard \& Dickson, 1997).

First, several relevant learning concepts are briefly described to place them in theoretical context, then their application is illustrated in components of a specific educational software tool. 


\section{Stimulus discrimination and generalisation}

When we learn something about a specific event we may behave differently to that event than to others like it (discrimination), but we may also behave similarly to similar events (generalisation). Various instructional variables affect the balance between discrimination and generalisation during learning (Schwartz \& Reisberg, 1991). Scaffolding can use these variables to influence this balance, so as to make learning more efficient (Etzel \& LeBlanc, 1979). A child learning letter identification needs to learn to differentiate between the letters (discrimination), but must learn to treat as the same different fonts and sizes of each letter (generalisation).

\section{Response generalisation and shaping}

When we learn to make a particular response, we also acquire a tendency to make other responses similar to the learned one. This principle, response generalisation, can be used in scaffolding to help the learner make a desired response that is difficult. The scaffolding procedure gradually changes the response requirement from an easy (or more likely) response, through intermediate steps, until the desired difficult response is attained. This process is referred to as response shaping (Becker, Engelmann, \& Thomas, 1975). A child learning to print a word starts out with a rough approximation that might just barely be recognisable, but progresses with tutoring toward a more accurate rendition.

\section{Incremental or graduated change}

Discrimination, generalisation and shaping occur along conceptual dimensions of change (eg. size, form, color, delay, location, direction, etc). The scaffolding procedure must determine the dimensions that will be used in learning. It must also use increments of change that are not so large as to cause errors by preventing generalisation but not so small as to be inefficient (Mosk \& Bucher, 1984). For example, large changes in font or size can disrupt a child's prior learning to identify letters, but small changes can be tolerated.

\section{Interactive adjustment of instructional parameters}

Increments of change in events or response requirements used for scaffolding can be individualised by making them dependent on the learning process. If a small change in an event or a response requirement results in a learner error, that change can be reversed. Such interactive feedback loops in the instructional program are the means by which a program can respond to individual differences in learning profiles of different learners. 
A familiar concept seen in digital interactive games is the availability of a tier of performance levels from which players can choose a level appropriate to their self assessment of their skill levels. Interactive adjustment in scaffolding goes beyond this concept, in that the current level of expected performance is based on the system's ongoing assessment of the 'player's' performance and is adjusted dynamically on the basis of each response the 'player' makes (Touchette \& Howard, 1984). For a child learning to read a given word, a tutor's prompt (supply the word name) can be given after a delay that is increased or decreased according to a rule.

\section{Intra- and extra-stimulus visual prompts}

Visual prompts are elements that can be added to a display to influence discrimination and generalisation. As part of a scaffolding process they can be progressively added, removed, advanced or delayed. They can be closely integrated with the event they are prompting (intra-stimulus) or they can be relatively remote (extra-stimulus) (Schreibman, 1975). In general, intra-stimulus prompts are more effective. A child learning letter identification can be helped to attend to the distinctive features of each letter by highlighting these in some way, such as color accenting. Coloring a letter element creates an intra-stimulus prompt, whereas coloring the background creates an extra-stimulus prompt.

\section{Enhancement and blocking}

Some prompts can enhance the likelihood that a learner will learn to attend to the desired event when it is no longer prompted (enhancement). For example, an intra-stimulus prompt can highlight and direct attention to a critical feature of a learning task. Importantly, some prompts can interfere with the desired course of learning (blocking). For example, illustrations used as extra-stimulus prompts in children's first reading books can delay learning to read the words that accompany the illustration (Solman, Singh, \& Kehoe, 1992).

\section{Errorless learning}

Errorless learning refers to learning resulting from the use of instructional formats that use scaffolding procedures such as generalisation, discrimination, shaping and prompts to minimise errors by the learner during the learning process. An important characteristic of errorless learning formats is that, as well as providing effective and efficient instruction, they create a positive emotional context for learning that sustains interest and enthusiasm (Beale, 2000; Touchette \& Howard, 1984). 


\section{Prerequisite skills or knowledge}

All learning contexts build on skills that have been previously learned. According to the direct instruction model, it is important that a check is made for mastery of assumed skills or knowledge before new teaching commences (Wolery et al., 1992). For many children, especially those with specific learning disabilities, training to read words is more efficient when it is built on an existing ability to identify individual letters, than otherwise (Beale, 1998).

\section{Immediate positive feedback}

In a new learning task, immediate feedback for the learner's responses will promote efficient learning and motivation to continue. Feedback is seen as having an informational function and a motivational function. Scaffolding provides for the progressive removal of the immediacy of feedback as learning progresses. This can be valuable for promoting generality of learning by the introduction of a more natural context for maintaining the learned behaviour (Peterson, 1982; Stokes \& Baer, 1977). The ability of educational software to accurately deliver a complex schedule of feedback far exceeds that of a human tutor (Lepper \& Malone, 1987).

\section{Motivation to learn}

Analyses of theory and research on the role of motivation in learning indicate that, in general, higher motivation leads to better learning (Lepper \& Malone, 1987). The emergence of learning and success on the learning task may provide increasing intrinsic motivation as learning continues. Scaffolding can increase intrinsic motivation by reducing errors and maximising success. It can also provide for progressive reduction in external motivation as a task is learned (Barlow \& Hersen, 1984).

\section{Cognitive loading}

Research on human information processing during learning has shown that the capacity of a learner to process information is limited. There are ways to design a learning task to reduce this processing requirement, especially in the initial stages when none of the components of the task have become automatic (Kalyuga, 2000; Moreno \& Mayer, 2000). For example, scaffolding can be used to progressively increase cognitive load as the learner masters the learning task by progressively increasing the short term memory requirement of a component skill or by shifting instructional content between sensory modalities. 


\section{Integrated assessment}

Scaffolding is achieved in intelligent learning environments by the use of programming algorithms, combinations of logic rules and simple mathematical equations that adjust the future learning environment according to how the learner responds to the current environment. These algorithms monitor the interaction between the learner and the environment and use that information to decide what adjustments to make to the environment to maximally facilitate learning. This ongoing, dynamic process collects information about the course of learning that can be construed as integrated assessment of the learner's progress on the skills being taught. The term 'integrated' is used in preference to 'continuous' to avoid confusion with the concept of continuous assessment central to experimental and applied behaviour analysis (Barlow \& Hersen, 1984; Nelson \& Hayes, 1981), in which continuous assessment refers to repeated, but periodic, measurement of the status of a behaviour. The term 'integrated' better captures the interactive monitoring that is an integral component of the scaffolding algorithms. Unlike traditional assessment concepts, integrated assessment is not simply assessment of the learner, but rather assessment of the learner's response to the instructional environment. As such it places emphasis on the adequacy of the environment to instruct the learner, as distinct from a static conception of the learner's current ability or aptitude (Nelson \& Hayes, 1981). The information collected by this monitoring process can be recorded by the program for automatic analysis and made available to the learner and the educator in the form of appropriate graphs that summarise the learner's progress.

\section{Scaffolding principles applied in an intelligent learning environment}

How these learning principles are applied to create scaffolding in specific intelligent environments depends on many considerations, including the nature of the content, the learning goals, and the developmental level and other characteristics of the potential learners who will use the environment. Instructional research on errorless learning and scaffolding has originated primarily in the context of efforts to develop a technology of learning for the prevention and remediation of learning failure in children with learning problems. The resulting technology has since been widely applied with considerable success to the learning of academic, vocational, self care, communication and social skills in children and adults, both with and without identified learning disabilities (Beale, 2000; Schreibman, 1975; Touchette \& Howard, 1984; Wolery et al., 1992). Most of these applications concern learning environments involving human instructors, however. 
Perhaps because the development of appropriate digital systems has been quite recent, similar applications to intelligent learning environments have been slow to appear. Even in areas of academic learning that have been a focus for research on the use of scaffolding by human teachers, such as reading and mathematics, descriptions of digital applications of scaffolding technology are hard to find.

The following sections present examples of how scaffolding concepts have been integrated into an intelligent learning environment designed to teach basic reading skills to young children with learning disabilities. The software application [1], which has yet to be evaluated in clinical trials, represents an experiment on the integration of scaffolding strategies into basic skills educational software. The individual scaffolding strategies used in the software have been subject to prior testing with human tutors and, in some instances, also with computerised tutoring (Beale, 2000). Although the curriculum content and target population are quite specific, these examples might serve as useful models for others involved in the design of learning software. In particular, it is possible to follow the process of translating a scaffolding concept into a software design strategy. These examples are not being offered here as models of best practice; there may well be better alternatives found in the future. Rather, they are offered as an illustration of a working model based of the application of learning principles into software to create an intelligent learning environment.

This outline does not provide a full picture of the use of scaffolding concepts and strategies in the software, because it describes only one of a set of integrated activities. Some of the strategies are incorporated across activities, rather than just within activities. The individual activities are located at sites in a 3-D virtual city, which the learner (player) can navigate freely or with guidance from a virtual robot.

The activity described here is called 'Balloon Targets'. Its objective, shared partly with some other activities, is to teach the young player to identify, when they are named, the lower case letters b, d, p, \& q. The main interface for this activity is shown in Figure 1. In this activity a full screen, upper level room is displayed. The player looks at a wall with a window out to a cityscape. Balloons traverse this window, left to right. Each balloon may have a letter (b, $d, p$, or $q)$ displayed on it. As a balloon passes the window it can be hit by a target moved by the player, but only if the player has first clicked on same letter as displayed on the balloon, or named by the voice. As each balloon appears at the left of the window, the name of a letter is spoken is spoken. When a balloon is hit, it flashes a color (red, white or blue) corresponding to the accuracy of the shot. At first the player is guided (prompted) to choose the correct letter. This guidance is gradually removed as the player makes correct choices, but it is gradually restored if 
the player makes mistakes. As well as guidance, correction is provided whenever the player clicks on an incorrect letter. The parameters chosen for scaffolding strategies, including placement of visual prompts, prompt delay intervals and increments of change, are based as far as possible on prior research (Beale, 2000).

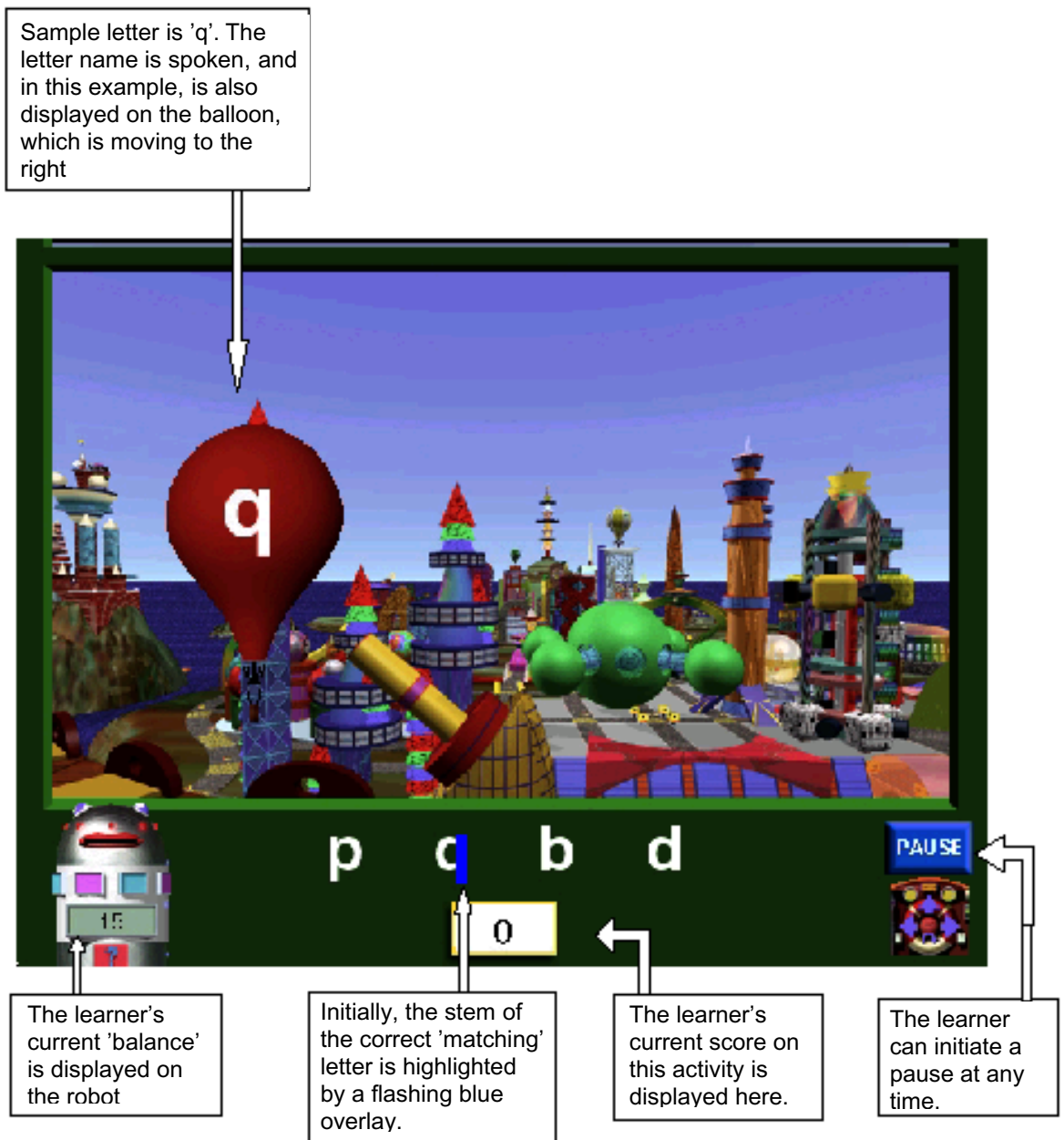

Figure 1: Balloon Targets: Main interface

About 20 percent of children continue to confuse the letters $b, d, p$ and $q$ in reading, spelling and writing after three or more years of schooling, and this impedes the development of more complex skills such as word 
analysis (Corballis \& Beale, 1983). Mastery of letter identification is widely regarded as prerequisite for more advanced reading skills. Although the 'balloons' activity does not explicitly require the learner to speak the letter name when shown the letter, it has been shown that the training given in this activity typically will have this result, an aspect of a phenomenon known as stimulus equivalence (Sidman, 1971).

The following sections list the scaffolding concepts that are used in the software application, and describe how they are represented as software design strategies. These specific concepts are derived from the more general concepts listed in a previous section of this article.

\section{Scaffolding concept: Minimise errors by ensuring that prerequisite skills are already mastered}

In most print fonts, $\mathrm{b}, \mathrm{d}, \mathrm{p} \& \mathrm{q}$ are simply left-right or up-down reflections of the same shape. In some fonts $\mathrm{q}$ has the added feature of an extra stroke on the foot of the vertical stroke. The learner must learn to distinguish between them on the basis of left-right and up-down differences, something which children have been shown to find particularly difficult, especially since their own left-right sense is not well established when they first enter school.

Reading words and sentences, not just letter discrimination, requires an ability to scan consistently from left to right, and this in turn requires the development in the learner of a left-right somatosensory gradient (a difference in feeling between the left and right sides of the body). The software application includes two compulsory activities that facilitate development of the left-right gradient. The activity 'Controlled Intersections' teaches left-right response differentiation, and 'Road Repairs' teaches left-right and up-down stimulus discrimination. These skills supplement those taught in 'Balloon Targets', and both include their own sets of scaffolding strategies.

\section{Controlled intersection}

This activity (Figure 2) teaches left/right response differentiation. The child learns to make left/right responses to instructions that contain no visual prompts. This skill is taught at intersections controlled by traffic lights. The buggy stops automatically for the red light. A spoken instruction is given to go straight ahead, turn left, or turn right. The child must learn to respond by clicking the correct direction control (straight ahead, left, or right). At first the child is automatically guided to make the correct turn. This guidance is gradually removed as the child makes correct choices, but it is gradually restored if the child makes mistakes. As well as guidance, 
correction is provided whenever the child attempts to make an incorrect choice. When the child clicks the correct turn control, the instruction (e.g., 'turn left') is repeated, and the control flashes green as the computer moves the buggy through the intersection.

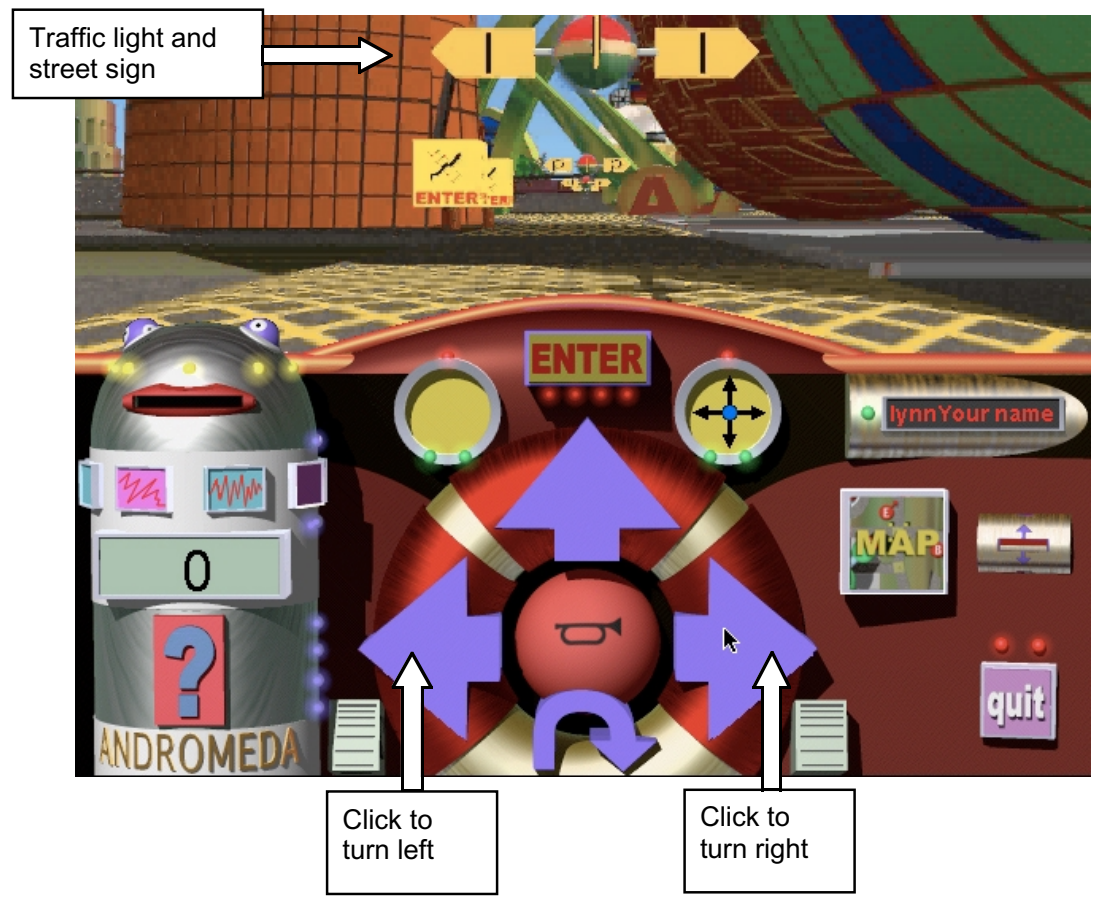

Figure 2: Controlled intersection

\section{Road repairs}

The child is taught awareness of the left/right and up/down dimensions, and to transpose differences on these dimensions onto objects in the immediate environment (Figure 3). This skill is taught in any street. The buggy stops automatically when it encounters an unpaved hole in a street. The computer displays a full screen view from above of the hole and the pavers (blocks) that must be placed to repair it. When the pavers are correctly placed, the screen returns to the 'buggy view' and the buggy can proceed to the next intersection. The activity teaches the child to distinguish between different orientations of the same basic shape. The pavers are all 'L-shaped', but in 4 different orientations, corresponding to faint paver outlines (templates) in the hole to be paved. The child must drag and drop each paver to the correct shaped hole. At first the child is guided (prompted) to make a correct drop. This guidance is gradually 
removed as the child makes correct choices, but it is gradually restored if the child makes mistakes.

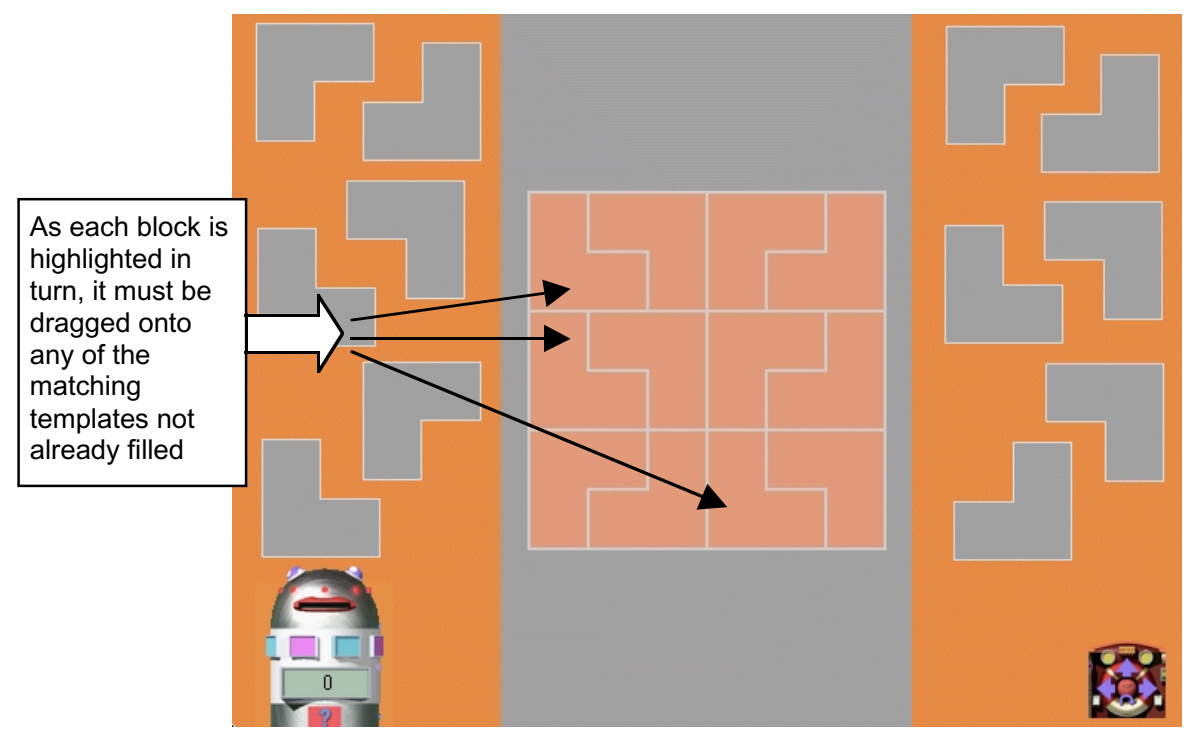

Figure 3: Road repairs

\section{Scaffolding concepts: Maximise motivation and provide immediate feedback}

Participation in the activity is either selected by the learner from a menu of options (using a pop up map), or selected on a whim when driving past the activity site. This element of choice (self selection) is intended to facilitate motivation to learn. A game format (using balloons as targets) is used because it was considered to be a fun activity for children, providing intrinsic motivation to use the activity.

Correct actions by the learner are immediately rewarded with positive feedback. For example, clicking on the correct letter immediately stops the balloon and produces the target. This is marked by auditory and visual feedback. Correct actions are immediately rewarded by points accumulated on an on screen display. These points may be exchanged later for tangible rewards displayed in a virtual shop. Incorrect actions are immediately corrected and the correct action is prompted.

Additional extrinsic reward is available to the learner in the form of charts showing improvement in skills over time. These may be printed out and stuck on the wall. Motivation is maintained by minimising errors during 
learning. It has been shown that error minimisation and maximisation of success are important for maintaining motivation for specific learning tasks and for learning generally (Lepper \& Malone, 1987). The strategies used for this are described in the following section.

\section{Scaffolding concept: Errorless learning}

At first, the sample letter $(b, d, p, q)$ is both named and displayed (immediately) on the balloon. This means that the learner has only to match the display on the balloon with one of the letters shown below the window. This learning format is called visual simultaneous matching to sample, the easiest applicable learning procedure. As learning progresses, the visual display on the balloon is progressively delayed, allowing the emergence of delayed matching to sample. This more difficult for the learner than the simultaneous procedure, so it is achieved in small transitional steps in which the delay is progressively increased or decreased according to whether the learner makes a correct or incorrect choice on the previous attempt. This procedure, called progressive delayed prompting, has been shown to maximise the efficiency of learning and decrease the likelihood of errors during learning (Touchette \& Howard, 1984)

Progressively, the task changes from simultaneous matching to sample, through delayed matching to sample, to a simple identification task in which the learner is able to respond to hearing a letter name spoken, by choosing the correct letter from the display of four alternative letters $(b, d$, $\mathrm{p}, \mathrm{q})$. To further assist the learner with this task, the learner may at first be shown which is the correct 'target' letter by 'flashing' part of the correct letter. The letter stem is flashed, because the position of the letter stem (left, right, up, down) is what distinguishes the four letters from one another. This prompting procedure, called highlighting a distinctive feature, helps the learner make a correct selection (Schreibman, 1975). Later in learning, the highlighting is progressively delayed as described previously, to assist the learner to choose correctly without the aid of the highlighting.

Highlighting, as used here, provides an example of scaffolding using an intra-stimulus prompt. These prompts have been shown to be the most efficient method of drawing the learner's attention to the important features of the task (Schreibman, 1975).

Problems with sustaining attention to task may be minimised by maximising motivation to participate and learn. This is addressed by the interactive features of the activity, such as immediate feedback on actions, including positive feedback for correct actions, immediate correction of errors, repeating of instructions where required, and demonstration (modeling) of required actions. Instructions and modeling are scaffolding 
features because they are provided when required at the beginning of training, but are subsequently available only on request by the learner.

Problems with selective attention are addressed by requiring the learner to manipulate the letters and other important objects to be learned (Beale, 2000). In the 'Balloon Targets' activity, this strategy is used to direct the learner's attention to the letters to be learned and their distinctive features.

\section{Scaffolding concept: Cognitive loading}

Learners vary in their ability to hold information in working memory during the task itself and subsequently to retrieve information from long term memory. In particular, children with learning disabilities or hyperactivity often have working memory problems that seem to be compounded by a lack of effective strategies for structuring learning tasks to minimise the impact of their memory problems (Beale, 1998).

In the current example, an attempt has been made to minimise the impact of memory problems by using tasks with a very low initial working memory requirement and then gradually increasing this as learning progresses. In the 'Balloon Targets' activity, the initial requirement to remember the sample letter is almost zero, since the visual sample is displayed immediately and remains available until the correct action has been performed. The sample and the target array of letters are separated only by a small visual angle that allows simultaneous scanning. When, during progressive delayed prompting, a delay is introduced between the letters and then increased, the process is gradual and should therefore support a gradual strengthening of the relevant process in working memory.

\section{Scaffolding concept: Readiness for information}

Most of the activities include pauses that are only terminated by an action by the learner, a procedure which guarantees that critical information is presented only on request from the learner, when the learner is ready. In addition, a 'pause' button allows the learner to introduce a self regulated timeout at any stage.

\section{Scaffolding concept: Instruction and modeling}

The activities provide two levels of instruction. Verbal instructions are automatically given and, if necessary, are repeated automatically twice before an appropriate action is modeled. Also, the learner can elicit full instructions for an activity at any time by clicking on the 'personal robot' that accompanies the learner in the navigation vehicle and on activities. 


\section{Integrated assessment in reading basics}

Data collected by the scaffolding algorithms are stored in arrays where they are analysed for display as charts intended to be understandable either to educators or parents (Figure 4: Adult level graph) or young children (Child version). Charts are accessed by the educator or learner from a screen outside the main program. This screen allows the person to select the player and the activity they wish to view the results for. To facilitate easy reading these charts present the minimum information required to show the child's initial performance as well as performance on the last three plays. These summary charts are intended to convey the most essential information as clearly as possible.

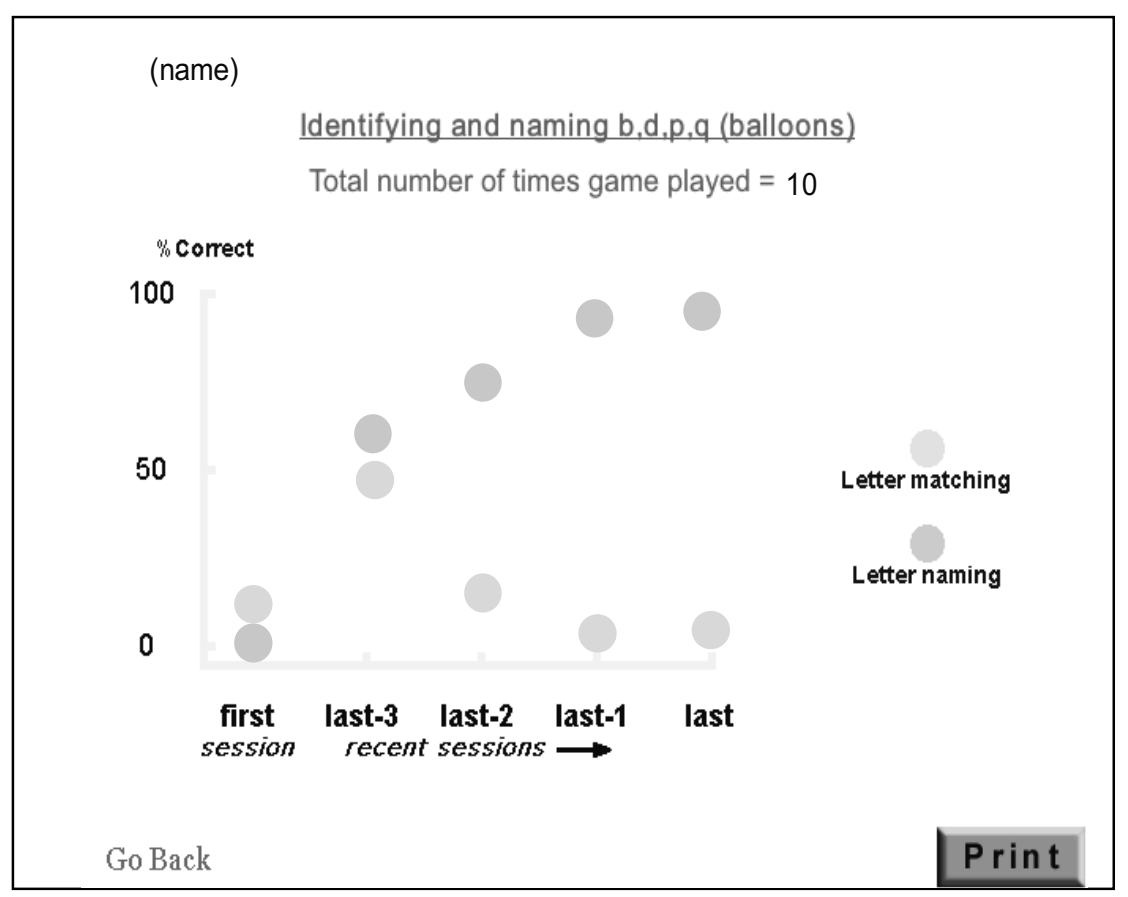

Figure 4: Adult level graph

The graphical record above shows the child's learning of two skills taught in the activity, letter matching (green) and letter naming (orange). Letter matching is the easier skill, choosing the letter beneath the window that matches the letter displayed on the balloon. Letter naming is more difficult, choosing the letter beneath the window that corresponds to the letter spoken, before it is displayed on the balloon. 
The strategy used in this activity is to first teach letter matching, then progressively introduce letter naming. As the child learns letter naming, opportunities for letter matching will gradually disappear and will only reappear if mistakes are made on attempts at letter naming.

In the example shown above, the child has used the activity ten times. On the first session, $10 \%$ of letter matching attempts were successful (green, $10 \%$ ) and letter naming was either not tried or not successful (orange, $0 \%$ ). By the last $\left(10^{\text {th }}\right)$ session, the child was able to correctly name the letter every time $(100 \%)$ and there were no opportunities for letter matching $(0 \%)$. Over the ten sessions on this activity, the child has first learned to match the letters (identify similarities and differences in shape), then has learned to associate each of the four letters with its spoken name.

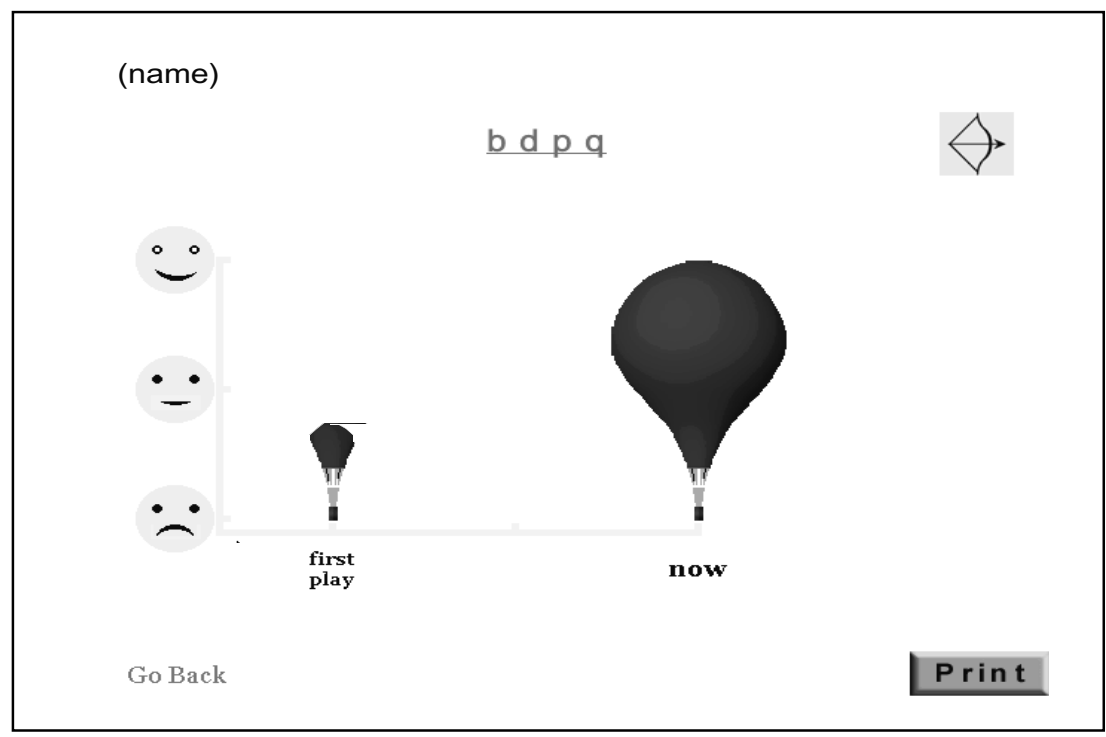

Figure 5: Child level graph

The child graph allows a young child to see more easily how performance has changed from the first use of the activity to now. The taller the balloon, the better the performance. The balloon and the icon at the top right corner will remind the child which activity the graph refers to.

\section{Conclusions and recommendations}

These examples of activities from a specific intelligent learning environment show that activities for learning provide numerous opportunities for applying scaffolding concepts that will support learning. 
The corresponding strategies used in this particular software are readily transferable to other learning environments using different content and with different learning goals, at least at a general level. Although the learning environment from which the current examples were taken was specifically designed for young children with learning problems, there is no reason why scaffolding strategies should not improve the learning efficiency software designed for other types of learner. The current drive in schools and tertiary education institutions to increase distance education resources and provide courses for less able learners could well result in a greater need for intelligent learning environments that could provide more effective instruction for students of widely varying ability. The scaffolding concepts and strategies described in this paper are an indication of the sort of development that will be required to meet this need.

All that is required to include scaffolding and integrated assessment into educational software is close collaboration between a learning expert with a good understanding of scaffolding and a software design team able to translate the scaffolding requirements into learning algorithms. Setting the parameters for algorithms, such as initial prompt delays and delay increments, is inevitably a 'seat of the pants' operation requiring personal experience in tutoring as well as familiarity with the relevant empirical literature on scaffolding design. Because the best solution is likely to depend on the content being taught and the specifics of the intelligent environment being used, it is advisable to design the software so that parameters can be varied during a testing program. These parameters should not be locked down before the software has been thoroughly tested and adjusted on the basis of responses from representative learners.

\section{Acknowledgement}

This research was conducted when the author was at HopeLab, Palo Alto, California. Preparation of this paper was funded by HopeLab.

\section{Endnote}

1. Copies of the software on $C D$ are available free for research purposes from the author. Email ibeale@psy.unsw.edu.au or ibeale@bigpond.com. Minimum system requirements are PC Pentium 1 or Apple OS 8 or later, 16 MB RAM, 8 x CD drive, MPC compatible sound card, 16 bit colour monitor.

\section{References}

Barlow, D. H. \& Hersen, M. (Eds) (1984). Single-case experimental design: Strategies for studying behavior change (2nd ed.). New York: Pergamon.

Beale, I. L. (1998). Learning disabilities. In A. Bellack \& M. Hersen (Eds), Comprehensive Clinical Psychology (Vol. 9, pp. 37-55). London: Pergamon. 
Beale, I. L. (2000). Integrated neural learning. Arrowtown, NZ: Brainwaves Limited.

Becker, W. C., Engelmann, S. \& Thomas, D. R. (1975). Teaching 2: Cognitive learning and instruction. Chicago: Science Research Associates.

Butler, D. L. (1998). In search of the architect of learning: A commentary on scaffolding as a metaphor for instructional interactions. Journal of Learning Disabilities, 31(4), 374-385.

Corballis, M. C. \& Beale, I. L. (1983). The ambivalent mind: The neuropsychology of laterality. Chicago: Nelson Hall.

Denning, R. \& Smith, P. J. (1998). A case study in the development of an interactive learning environment to teach problem-solving skills. Journal of Interactive Learning Research, 9(1), 3-36.

Dube, W. V., Iennaco, F. M., Rocco, F. J., Klederas, J. B. \& McIlvane, W. J. (1992). Microcomputer-based programmed instruction in identity matching to sample for persons with severe disabilities. Journal of Behavioral Education, 2(1), 29-51.

Etzel, B. C. \& LeBlanc, J. (1979). The simplest treatment alternative: appropriate instructional control and errorless learning procedures for the difficult-to-teach child. Journal of Autism and Developmental Disorders, 9, 361-382.

Hitchcock, C. (2001). Balanced instructional support and challenge in universally designed learning environments. Journal of Special Education Technology, 16(4). [verified 26 Apr 2005] http:/ /jset.unlv.edu/16.4/hitchcock/first.html

Judge, S. L. (2001). Computer applications in programs for young children with disabilities: Current status and future directions. Journal of Special Education Technology, 16(1). [verified 26 Apr 2005] http://jset.unlv.edu/16.1/Judge/first.html

Kalyuga, S. (2000). When using sound with a text or picture is not beneficial for learning. Australian Journal of Educational Technology, 16(2), 161-172. http://www.ascilite.org.au/ajet/ajet16/kalyuga.html

Kameenui, E. J., Simmons, D. C., Chard, D. \& Dickson, S. (1997). Direct-instruction reading. In S. A. Stahl \& D. Hayes (Eds), Instructional models in reading. (pp. 5984). Mahwah, NJ: Erlbaum.

Lepper, M. R., Drake, M. F. \& O'Donnell-Johnson, T. (1997). Scaffolding techniques of expert human tutors. In K. Hogan \& M. Pressley (Eds), Scaffolding student learning: Instructional approaches and issues (pp. 108-144). Cambridge, MA: Brookline Books.

Lepper, M. R. \& Malone, T. W. (1987). Intrinsic motivation and instructional effectiveness in computer-based education. In R. E. Snow \& M. J. Farr (Eds), Aptitude, learning, and instruction III. Conative and affective process analysis. Hillsdale, NJ: Erlbaum.

Moreno, R. \& Mayer, R. E. (2000). A learner-centered approach to multimedia explanations: Deriving instructional design principles from cognitive theory. Interactive Multimedia Electronic Journal, 2(2). [verified 26 Apr 2005] http://imej.wfu.edu/articles/2000/2/05/index.asp

Mosk, M. D. \& Bucher, B. (1984). Prompting and stimulus shaping procedures for teaching visual-motor skills to retarded children. Journal of Applied Behavior Analysis, 17, 23-34. 
Nelson, R. O. \& Hayes, S. C. (1981). Nature of behavioral assessment. In M. Hersen \& A. S. Bellack (Eds), Behavioral assessment: A practical handbook (2 ed., pp. 3-37). New York: Pergamon.

Peterson, N. (1982). Feedback is not a new principle of behavior. The Behavior Analyst, 5, 101-102.

Reid, D. K. (1998). Scaffolding: A broader view. Journal of Learning Disabilities, 31(4), 386-396.

Sampson, D., Karagiannidis, C. \& Kinshuk (2002). Personalised learning: Educational, technological and standardisation perspective. Interactive Educational Multimedia, 4, 24-39. [verified 26 Apr 2005] http://www.ub.es/multimedia/iem/down/c4/Personalised_Learning.pdf

Schreibman, L. (1975). Effects of within-stimulus and extra-stimulus prompting on discrimination learning in autistic children. Journal of Applied Behavior Analysis, 8, 91-112.

Schwartz, B. \& Reisberg, D. (1991). Learning and memory. New York: W. W. Norton.

Scruggs, T. E. \& Mastropieri, M. A. (1998). What happens during instruction: Is any metaphor necessary? Journal of Learning Disabilities, 31(4), 404-408.

Sidman, M. (1971). Reading and auditory-visual equivalences. Journal of Speech and Hearing Research, 14, 5-13.

Solman, R. T., Singh, N. N. \& Kehoe, E. J. (1992). Pictures block the learning of sightwords. Educational Psychology, 12, 143-153.

Stokes, T. F. \& Baer, D. M. (1977). An implicit technology of generalization. Journal of Applied Behavior Analysis, 10, 349-367.

Touchette, P. E. \& Howard, J. S. (1984). Errorless learning: Reinforcement contingencies and stimulus control transfer in delayed prompting. Journal of Applied Behavior Analysis, 17, 175-188.

Wolery, M., Ault, M. J. \& Doyle, P. M. (1992). Teaching students with moderate to severe disabilities. White Plains, New York: Longman.

Wong, B. Y. (1998). Analyses of intrinsic and extrinsic problems in the use of the scaffolding metaphor in learning disabilities intervention research: An introduction. Journal of Learning Disabilities, 31(4), 340-343.

Wood, D. J., Bruner, J. S. \& Ross, G. (1976). The role of tutoring in problem solving. Journal of Child Psychology and Psychiatry, 17, 89-100.

Ivan L. Beale is Visiting Associate Professor, School of Psychology, University of New South Wales, Sydney. Tel: +61 0401918395

Email: ibeale@psy.unsw.edu.au, ibeale@bigpond.com 